\title{
Dynamics of the Jet Stream
}

\author{
by \\ T. Sato \\ Meteorological Research Institute \\ (Received April 11, 1951)
}

Contents
Abstract
1. Characteristics of the Jet Stream
2. Integration of the Equations of Stationary Motion
3. Surface of Discontinuity
4. Mixing Layer
5. Existence of the Jet Stream
6. Structure of the Jet Stream
7. On the Mechanism of the General Circulation
Acknowledgement
References

\begin{abstract}
In discussing the structure of typhoon before, the author pointed out the existence of a maximum of wind velocity, that is, a sort of jet stream, just at the outer side of the tropopause funnel which forms the eye of typhoon. The jet stream associated with the general circulation of the atmosphere seems essentially to be the same as that with the typhoon. Therefore the author tried to explain the phenomenon, adopting the following four assumptions: (A) stationary, (B) uniform in one direction, (C) frictionless, (D) autobarotropic, and deriving integrals from the initial equations of motion including inertia terms, in the same way as in the previous paper. The integrals represent respectively the law of conservation of (angular) momentum, vorticity and energy.

From the law of conservation of energy can be derived an, equation which determines the form of the surface of discontinuity. The surface inclines the steeper, the smaller the temperature difference and the larger the wind velocity difference between the both sides of the surface. In the neighbourhood of the surface, however, develops a mixing layer at the same time owing to the discontinuity of wind velocity. The thickness of
\end{abstract}


the layer becomes the larger, the smaller the temperature difference and the larger the wind velocity defference, that is, the steeper the surface inclines, the larger the mixing layer becomes in thickness.

From the law of conservation of vorticity it results that there must exist convective motions in the meridional section. of the atmosphere. Now, air particles displacing northward get extremely stronger in west wind velocity as they approach the axis of the earth, as momentum is conserved along a streamline in an air mass. The strong wind thus developed draws down the surface of discontinuity lying above it and draws up that lying beneath. It follows thus that air particles can not proceed on their way over some extent northward, owing to the barrier which they form themselves. In this way, the meridional circulation of the troposphere presents necessarily a cellular structure, and the air in lower latitudes can not arrive directly at the pole. On the other hand, the effect of friction is the most intense and the mixing layer becomes the largest at the northern end of the cell thus formed. The exchange of air between lower and higher latitudes is carried out only through friction. Thus the jet stream is developed in the northern part of each cell, that is, at the northern end of the southern side of the mixing layer.

The structure of the jet stream thus derived is shown in the figures. These figures, which are derived theoretically, coincide very well with those analysed hitherto by many investigators.

\section{Characteristics of the det Stream}

In recent years, abundant interesting investigations were done on the jet stream $[1 \sim 6]$. According to them, the outstanding characteristics of the jet stream seem to be as follows:

(1) The jet stream appears in a wide extent from about $20^{\circ} \mathrm{N}$ to about $70^{\circ} \mathrm{N}$.

(2) The situation of the jet fluctuates seasonally. It deviates northward in summer and southward in winter [7].

(3) The jet stream is stronger in winter than in summer [7].

(4) The jet stream appears in the upper part of the troposphere, at the altitude of $10-15 \mathrm{~km}$ above the ground.

(5) The wind velocity is meximum at the center of the jet, from where it decreases rapidly to the south as well as to the north. The decrease southward from the center is represented a.lmost by the constancy of absolute angular momentum. and the decrease northword almost by the constancy of absolute vorticity $[8 \sim 10]$. 
(6) The wind velocity decreases slowly both upward and downward from the center of the jet. Above the center of the jet, the position of maximum wind velocity shifts itself southward from the center, and below the center also it shifts southward. [8 10]

(7) The maximum wind velocity exceeds $100 \mathrm{~m} / \mathrm{s}$ frequently.

(8) Isobaric surfaces in the neighourhood of the jet incline northward. The inclination is the steepest at the center of the jet, from where it decreases both rorthward and southward.

(9) Isothermal surfaces hang down northward in the southern half part of the jet region. They stretch upward for some extent (about $10^{\circ}$ latitude) northward from the center of the jet, and then hang down again slowly in the further extent [8 10].

(10) Under the center of the jet, there exists a surface of discontinuity hanging down southward.

(11) In the neighbourhood of the center, there exists a surface of discontinuity hanging down northward. This surface seems to be fairly difficult to analyse [8 10].

(12) Fronts in the neighbourhood of the jet seem to be indistinct and often be in fragments or multiple $[8 \sim 10]$.

(13) The jet situates just over or a little northward over the frontal layer at the earth's surface. In other words, it situates nearly ove the ground where isothermal lines are the most dense.

(14) In the troposphere are observed usually two or more jets, the one about $15^{\circ}$ lat. north-(or south-) ward distant from the other. One jet separates frequently into two or more jets, which often confluent inversely into one. In those cases, the southern jet situates higher in general [7].

(15) The jet is not always constantly situated, but it often moves slowly south- or northward. The southward movement is fairly systematic and especially well observed [7], [11].

(16) The jet stream surrounds the earth nearly along a latitude line and moves often eastward in a wavelike motion [7].

(17) The jet stream seems to be connected with the planetary wave and with perturbations generated in it.

A perfect theory on the jet stream must explain systematically all the above mentioned characteristics. As, however, it is very difficult to construct such a theory, we will treat here only of the structure of the jet stream under the assumption of stationary motion and touch the mechanism of the general circulation associated with i.t.

\section{Integration of the Equations of Stationary Motion}

We adopt the following assumptions in integrating the equations of motion:

(A) motion is stationary,

(B) motion is uniform in a direction,

(C) fluid is frictionless,

(D) fluid is autobarotropic. 


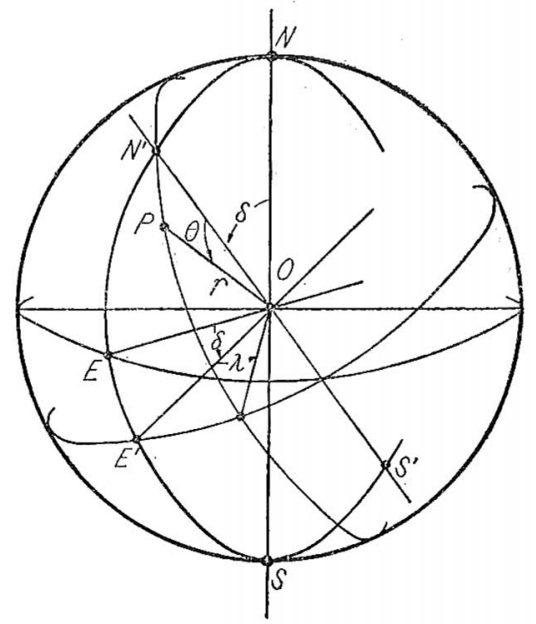

Fig. 1 Axis of Coordinates.

As papers are already published $[12 \sim 14]$ concerning the fluid motion under these assumptions, the calculations will be treated here only in abbreviation. If we adopt spherical coordinates $(r, \theta, \lambda)$ on the basis of an axis $\mathrm{ON}^{\prime}$ (Fig. 1), inclined by an angle $\delta$ from the earth's axis $\mathrm{ON}$, then the components of the angular velocity vector $\omega$ of the earth are

(1 a) $\omega_{r}=\omega(\cos \delta \cos \theta-\sin \delta \sin \theta \cos \lambda)$,

(1 b) $\omega_{\theta}=-\omega(\cos \delta \sin \theta+\sin \delta \cos \theta \cos \lambda)$,

(1c) $\omega_{\lambda}=\omega \sin \delta \sin \lambda$.

Therefore, the equation of motion

$$
\frac{\partial \boldsymbol{v}}{\partial t}+(\boldsymbol{v} \cdot \nabla) \boldsymbol{v}+2 \omega \times \boldsymbol{v}+\nabla \phi+s \nabla p=0
$$

is expressed under the above assumptions (A), (B), (C) and (D), as

(2a) $\quad v^{\prime} \cdot \frac{\partial v_{r}}{\partial r}+\frac{v_{\theta}}{r} \frac{\partial v_{r}}{\partial \theta}-\frac{v_{\theta}^{2}+v^{2}}{r}-2 \omega \cos \delta \sin \theta \cdot v+g+s \frac{\partial p}{\partial r}=0$,

(2b) $v_{r} \cdot \frac{\partial v_{\theta}}{\partial r}+\frac{v_{\theta}}{r} \frac{\partial v_{\theta}}{\partial \theta}+\frac{v_{r} v_{\theta}}{r}-\frac{\cot \theta}{r} v^{2}-2 \omega \cos \delta \cos \theta \cdot v+\frac{s}{r} \frac{\partial p}{\partial \theta}=0$,

(2c) $\quad v_{r} \frac{\partial v}{\partial \theta}+\frac{v_{\theta}}{r} \frac{\partial v}{\partial \theta}+\frac{\cot \theta}{r} v_{\theta} v+\frac{v_{r} \cdot v}{r}+2 \omega \cos \delta\left(\sin \theta \cdot v_{r}+\cos \theta \cdot v_{\theta}\right)=0$.

It must be noticed here that all the dependent variables must not contain $\lambda$, because the motion is considered to be uniform in the $\lambda$-direction. This can be said also for $\omega$, therefore
$\left(1 \mathrm{a}^{\prime}\right)$
$\omega_{r}=\omega \cos \delta \cos \theta$,
$\left(1 \mathrm{~b}^{\prime}\right)$
$\omega_{\theta}=-\omega \cos \delta \sin \theta$,
$\left(1 c^{\prime}\right)$
$\omega_{\lambda}=0$

are used instead of (1) in (2). The treatment of our problem is therefore correct only in the extent that the values $\left(1^{\prime}\right)$ can be used instead of (1). For instance, in the north-south motion of air in lower latitudes, $\delta \rightarrow \frac{\pi}{2}$ and $\lambda \rightarrow \frac{\pi}{2}$; therefore $\omega_{\lambda} \risingdotseq \omega$. In those cases, it is unreasonable to use the values ( $\left.1^{\prime}\right)$ instead of (1). As we mainly treat zonal motions only in higher latitudes, it is sufficiently permissible to use ( $\left.1^{\prime}\right)$ instead of (1), for, if $\delta$ is sufficiently small in (1), then we get $\left(1^{\prime}\right)$.

From the equation of continuity

$$
\frac{\partial}{\partial r}\left(r^{2} \sin \theta \cdot r \cdot r\right)+\frac{\partial}{\partial \theta}\left(\frac{r \sin \theta \cdot v_{\theta}}{s}\right)=0
$$

it will be easily seen that $v_{r}$, $v_{\theta}$ can be expressed by employing the stream-function $\psi$ of $\left(\frac{v_{r}}{s}, \frac{v_{z}}{s}\right)$ as 


$$
v_{r}=-\frac{s}{r^{2} \sin \theta} \frac{\partial \phi}{\partial \theta^{\prime}}, \quad v_{\theta}=\frac{s}{r \sin \theta} \frac{\partial \psi}{\partial r} .
$$

Employing this function $\psi$ instead of $v_{r}$ and $v_{z}$, we get immediately from equation $(2 \mathrm{c})$

$$
\frac{\partial \psi}{\partial r}\left(\frac{1}{r} \frac{\partial v}{\partial \theta}+\cot \theta \cdot \frac{v}{r}+2 \omega \cos \delta \cos \theta\right)-\frac{1}{r} \frac{\partial \psi}{\partial \theta}\left(\frac{\partial v}{\partial r}+\frac{v}{r}+2 \omega \cos \delta \sin \theta\right)=0 .
$$

Putting

$$
V=r \sin \theta \cdot v+\omega \sin \delta \cdot r^{2} \sin ^{2} \theta,
$$

we get from the above equation

$$
\left(\frac{\partial \psi}{\partial r} \frac{\theta}{\partial \theta}-\frac{\partial \psi}{\partial \theta} \frac{\partial}{\partial r}\right) V=0
$$

from which we can get the following integral

$$
r \sin \theta \cdot v+\omega \cos \theta \cdot r^{2} \sin ^{2} \theta=V(\psi),
$$

where $V(\psi)$ is an arbitrary function of $\phi$ only.

the equation (4) expresses the law of conservation of angular momontum along a streamline in the meridional section including ON!' Thus it may be called a momentum equation.

Next, calculating $\frac{1}{r}\left\{\frac{\partial}{\partial r}[(2 b) \times r]-\frac{\partial}{\partial \theta}(2 a)\right\}$, we get

$$
\begin{gathered}
\left(v_{r} \frac{\partial}{\partial r}+\frac{v_{\theta}}{r} \frac{\partial}{\partial \theta}+\frac{\partial v_{r}}{\partial r}+\frac{v_{r}}{r}+\frac{1}{r} \frac{\partial v_{\theta}}{\partial \theta}\right)\left(\frac{\partial v_{\theta}}{\partial r}+\frac{v_{\theta}}{r}-\frac{1}{r} \frac{\partial v_{r}}{\partial \theta}\right) \\
+2\left(\frac{v}{r}+\omega \cos \partial \sin \theta\right)\left(\frac{1}{r} \frac{\partial v}{\partial \theta}-\cot \theta \frac{\partial v}{\partial r}\right)=0 .
\end{gathered}
$$

Calculating $\left(v \cdot \frac{\partial}{\partial r}+\frac{v_{\theta}}{r^{r}} \frac{\partial}{\partial \theta}\right)\left\{\frac{s}{r \sin \theta}\left(\frac{\partial v_{\theta}}{\partial r}+\frac{v_{\theta}}{r^{r}}-\frac{1}{r} \frac{\partial v_{r}}{\partial \theta}\right)\right\}$ and applying (4) and (3), we can derive the following equation

$$
\begin{gathered}
\left(\frac{\partial \psi}{\partial r} \frac{\partial}{\partial \theta}-\frac{\partial \psi}{\partial \theta} \frac{\partial}{\partial r}\right)\left\{\frac{s^{2}}{r^{2} \sin ^{2} \theta}\left(\frac{\partial^{2} \psi}{\partial^{2} r}+\frac{1}{r^{2}} \frac{\partial^{2} \psi}{\partial \theta^{2}}+\frac{1}{s} \frac{\partial s}{\partial r} \frac{\partial \psi}{\partial r}+\frac{1}{s r^{2}} \frac{\partial s}{\partial \theta} \frac{\partial \psi}{\partial \theta}-\frac{\cot \theta}{r^{2}} \frac{\partial \psi}{\partial \theta}\right)\right. \\
\left.+\frac{V V^{\prime}}{r^{2} \sin ^{2} \theta}-\omega \cos \delta \cdot V^{\prime}\right\}=0
\end{gathered}
$$

from which we get the second integral

$$
\begin{gathered}
\frac{s^{2}}{r^{2} \sin ^{2} \theta}\left(\frac{\partial^{2} \psi}{\partial r^{2}}+\frac{1}{r^{2}} \frac{\partial^{2} \psi}{\partial \theta^{2}}+\frac{1}{s} \frac{\partial s}{\partial r} \frac{\partial \psi}{\partial r}+\frac{1}{s r^{2}} \frac{\partial s}{\partial r} \frac{\partial \psi}{\partial \theta}-\frac{\cot \theta}{r^{2}} \frac{\partial \psi}{\partial \theta}\right) \\
+\frac{V V^{\prime}}{r^{2} \sin ^{2} \theta}-\omega \cos \delta \cdot V^{\prime}=\Psi^{\prime}(\psi),
\end{gathered}
$$

where $\Psi^{\prime}(\psi)$ is an arbitrary function of $\psi$ only.

The equation (5) expresses the law of consevation of vorticity (the $\lambda$-component of) along a streamline in the meridional section ON.' Thus it may be called a vorticity equation.

Finally, modifying (2a) we can derive the following equation

$$
\frac{1}{2} \frac{\partial}{\partial r}\left\{\frac{s^{2}}{r^{2} \sin ^{2} \theta}\left(\frac{\partial \psi}{\partial r}\right)^{2}+\frac{s^{2}}{r^{4} \sin ^{2} \theta}\left(\frac{\partial \psi}{\partial \theta}\right)^{2}+\left(\frac{r}{r \sin \theta}-\omega \cos \delta \cdot r \sin \theta^{2}\right)^{2}\right\}
$$




$$
-\Psi^{\prime} \frac{\partial \psi}{\partial r}+g+s \frac{\partial p}{\partial r^{r}}=0,
$$

from which we can get the third integral

$$
\begin{gathered}
\text { (6) } \frac{1}{2}\left\{\frac{s^{2}}{r^{2} \sin ^{2} \theta}\left(\frac{\partial \psi}{\partial r}\right)^{2}+\frac{s^{2}}{r^{4} \sin ^{2} \theta}\left(\frac{\partial \psi}{\partial \theta}\right)^{2}+\left(\frac{V}{r^{2} \sin \theta}-\omega \cos \delta \cdot r \sin \theta\right)^{2}\right\} \\
+g r+\int^{p} s d p=\Psi(\phi) .
\end{gathered}
$$

The equation (6) expresses the lcw of conservation of energy along a streamine in the meridional section and coincides with the energy equation called BERNoulli's theorem.

Putting $\delta=0$ in the integrals (4), (5) and (6), we get

$$
\begin{gathered}
r \sin \theta \cdot v+\omega r^{2} \sin ^{2} \theta=V(\psi), \\
\frac{s^{2}}{r^{2} \sin ^{2} \theta}\left(\frac{\partial^{2} \psi}{\partial r^{2}}+\frac{1}{r} \frac{\partial^{2} \psi}{\partial \theta^{2}}+\frac{1}{s} \frac{\partial s}{\partial r} \frac{\partial \psi}{\partial r^{\prime}}+\frac{1}{s r^{2}} \frac{\partial s}{\partial \theta} \frac{\partial \psi}{\partial \theta}-\frac{\cot \theta}{r^{2}} \frac{\partial \psi}{\partial \theta}\right) \\
+\frac{V V^{\prime}}{r^{2} \sin ^{2} \theta}-\omega V^{\prime}=\Psi^{\prime}(\psi), \\
\frac{1}{2}\left\{\frac{s^{2}}{r^{2} \sin ^{2} \theta}\left(\frac{\partial \psi}{\partial r}\right)^{2}+\frac{s^{2}}{r^{4} \sin ^{2} \theta}\left(\frac{\partial \psi}{\partial \theta}\right)^{2}+\left(\frac{V}{r \sin \theta}-\omega r \sin \theta\right)^{2}\right\} \\
+g r+\int^{p} \operatorname{sd} d=\Psi(\phi) .
\end{gathered}
$$

These integrals represent the stationary motion symmetrical with respect to the earth's axis.

Now, as we can get the equations (4), (5) and (6) putting $\omega \cos \delta$ instead of $\omega$ into the equations $\left(4^{\prime}\right),\left(5^{\prime}\right)$ and $\left(6^{\prime}\right)$, it can be seen to be sufficient if we treat only of the equations $\left(4^{\prime}\right),\left(5^{\prime}\right)$ and $\left(6^{\prime}\right)$ hereafter.

For the motion not of so large scale, we can use Cartesian coordinates instead of spherical ones. Also in cases when a large scale motion is analysed synoptically, it is usual to represent the ground surface for convenience' sake with a horizontal straight line. In the papers referred in section 1, this method is also adopted. Therefore relations will be given here also in Cartesian coordinates.

If the origin is put on the earth's surface and the $v$-axis is directed to the north by an angle $\beta$ from the east, then the integrals corresponding to (4), (5) and (6) are

$$
\begin{gathered}
u-2 \omega \sin \theta \cdot y+2 \omega \cos \theta \cos \beta \cdot z=V(\psi), \\
\left(4^{\prime \prime}\right) \quad s{ }^{2} \frac{\partial^{2} \psi}{\partial y^{2}}+s^{2} \frac{\partial^{2} \psi}{\partial z^{2}}+s \frac{\partial s}{\partial y} \frac{\partial \psi}{\partial y}+s \frac{\partial s}{\partial z} \frac{\partial \psi}{\partial z} \\
+V^{\prime}(V+2 \omega \sin \theta \cdot y-2 \omega \cos \theta \cos \beta \cdot z)-2 \omega s \cos \theta \sin \beta=\Psi^{\prime}(\psi), \\
\left(6^{\prime \prime}\right) \frac{1}{2}\left\{s^{2}\left(\frac{\partial \psi}{\partial y}\right)^{2}+s^{2}\left(\frac{\partial \psi}{\partial z}\right)+(V+2 \omega \sin \theta \cdot y-2 \omega \cos \theta \cos \beta \cdot z)^{2}\right\} \\
+g z+\int^{p} s d p=\Psi(\psi),
\end{gathered}
$$

where $\theta$ is measured from the equator. 


\section{Sur face of Discontinuity}

In order to give the form of the vertical section of a surface of discontinuity, it is insufficient to apply only the assumption of autobarotropy which was used in deriving the integrals, for the form of the section is different according to the functional relation between $s$ and $p$. Therefore the assumption of polytropy will be applied here as one of the most common cases of autobarotropy.

The relation of polytropy is

$$
p s^{n}=p_{0} s_{0}{ }^{n}, \quad \text { where } n=\frac{g}{g-k \gamma},
$$

where $\gamma$ is the lapse-rate of temperaure. Thus we get

$$
\int^{p} s d p=\frac{1}{\sigma} p^{\frac{n-1}{n}}=\frac{1}{\sigma} p^{\frac{R}{g} \gamma},
$$

where

$$
\sigma=\frac{n-1}{n} \frac{q_{0}^{n}}{p_{0}}=\frac{n-1}{n} \frac{p_{0}^{n-1}}{R^{n} \bar{T}_{0}{ }^{n}}=\frac{R r}{g} p_{0} \frac{R r}{g-R \gamma}\left(R T_{0}\right) \frac{g}{g-R \gamma} .
$$

Using these relations, we can get the form of the vertical section of the surface of discontinuity in spherical coordinates

$$
\begin{aligned}
& \text { (11) }\left[\sigma_{1} \Psi_{1}-\sigma_{1} g r-\frac{\sigma_{1}}{2}\left\{\frac{s_{1}^{2}}{r^{2} \sin ^{2} \theta}\left(\frac{\partial \psi}{\partial r}\right)_{1}^{2}+\frac{s_{1}^{2}}{r^{4} \sin ^{2} \theta}\left(\frac{\partial \psi}{\partial \theta}\right)_{1}^{2}+\left(\frac{V_{1}}{r \sin \theta}-\omega r \sin \theta\right\}^{2}\right]^{r_{2}}\right. \\
& =\left[\sigma_{2} \Psi_{2}-\sigma_{3} g r-\frac{\sigma_{2}}{2}\left\{\frac{s_{2}^{2}}{r^{2} \sin ^{2} \theta}\left(\frac{\partial \psi}{\partial r}\right)_{2}^{2}+\frac{s_{2}^{2}}{r^{4} \sin ^{2} \theta}\left(\frac{\partial \psi}{\partial \theta}\right)_{2}^{2}+\left(\frac{r_{2}}{r \sin \theta}-\omega r \sin \theta\right)^{2}\right\}\right]_{1}^{r_{1}}
\end{aligned}
$$

As, however, $v_{r}=-\frac{s}{r^{2} \sin \theta} \frac{\partial \psi}{\partial \theta}$ and $v_{\theta}=\frac{s}{r \cdot \sin \theta} \frac{\partial \psi}{\partial r}$ are sufficiently small compared with $v=\frac{V}{r \sin \theta}-\omega r \sin \theta$, therefore the equation (11) can be expressed approximately

$$
\begin{aligned}
& \left\{\sigma_{1} \Psi_{1}-\sigma_{1} g r-\frac{\sigma_{1}}{2}\left(\frac{V_{1}}{r \sin \theta}-\omega r \sin \theta\right)^{2}\right\}^{r_{2}} \\
& =\left\{\sigma_{2} \Psi_{2}-\sigma_{2} g r-\frac{\sigma_{2}}{2}(r \sin \theta\right. \\
&
\end{aligned}
$$

In the case when the lapse-rates are equal $\left(r_{1}=r_{9}\right)$ in both layers contacting with each other on the surface of discontinuity, we get

$\left(11^{\prime \prime}\right) \quad\left(\sigma_{1}-\sigma_{2}\right) g r=\left(\sigma_{1} \Psi_{1}-\sigma_{3} \Psi_{3}\right)-\frac{1}{2}\left\{\sigma_{1}\left(\frac{V_{1}}{r \sin \theta}-(\omega) r \sin \theta\right)^{2}\right.$

$$
\left.-\sigma_{2}\left(r \frac{V_{2}}{\sin \theta}-\omega r \sin \theta\right)^{2}\right\} \text {. }
$$

In the case of Cartesian coordinates, the relation corresponding to the equation $\left(11^{\prime \prime}\right)$ is

$$
\begin{aligned}
& \omega^{2} \sin ^{2} \theta \cdot y^{2}-2 \omega \sin \theta \cos \theta \cos \beta \cdot y z+\omega^{2} \cos ^{2} \theta \cos ^{2} \beta \cdot z^{2} \\
& +\omega \sin \theta \cdot \frac{\sigma_{1} V_{1}-\sigma_{2} V_{3}}{\sigma_{1}-\sigma_{2}} \cdot y+\left\{\frac{g}{2}-\omega \cos \theta \cos \beta \cdot \frac{\sigma_{1} V_{1}-\sigma_{2} V_{2}}{\sigma_{1}-\sigma_{2}}\right\} z=0,
\end{aligned}
$$


where the origin is placed on the section of the surface with the earth.

the equation (12) expresses a parabola. Because, if we rotate the axis of coordinates by an angle $\varepsilon$, where

$$
\tan \varepsilon=\frac{\sin \theta}{\cos \theta \cos \beta},
$$

and remove the origin appropriately as is shown in Fig.2, then (12) beoomes [12]

(14) $\zeta^{2}=4 p \eta$,

where

$$
\text { (14 a) } \begin{aligned}
p= & -\frac{g \sin \theta}{8 \omega^{2}}\left(\sin ^{2} \theta\right. \\
& \left.+\cos ^{2} \theta \cos ^{2} \beta\right)^{-\frac{3}{2}} .
\end{aligned}
$$

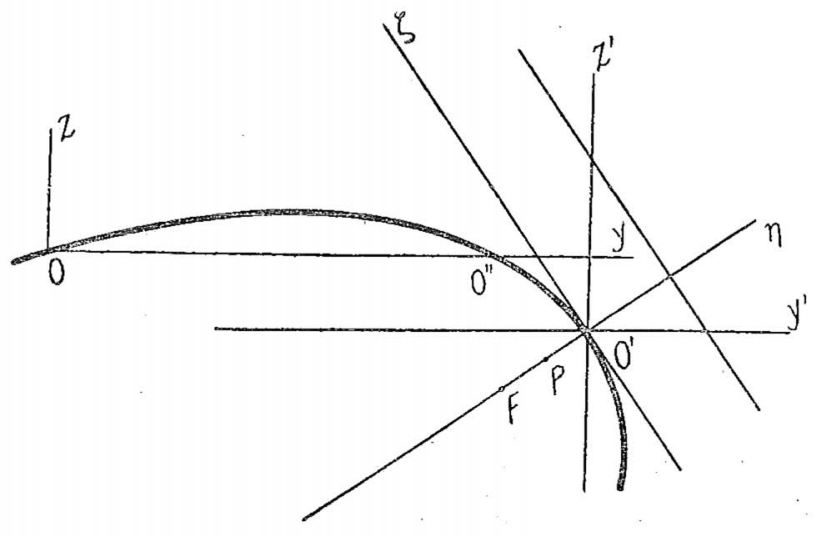

Fig. 2 Surface of Discontinuity.

The position $(Y, Z)$ of the origin $O$ of the initial coordinates $(y, z)$ seen from the system of coordinates $(\eta, \zeta)$ is after the above transformation,

(15 a)

$$
Y=-\frac{\left\{\frac{g}{2} \cos \theta \cos \beta\left(\sin ^{2} \theta+\cos ^{2} \theta \cos ^{2} \beta\right)^{\frac{1}{2}}-\omega \frac{\sigma_{1} V 1-\sigma_{2} V_{2}}{\sigma_{1}-\sigma_{2}}\left(\sin ^{2} \theta+\cos ^{2} \theta \cos ^{2} \beta\right)^{\frac{3}{2}}\right\}^{2}}{2 \omega^{2} g \sin \theta\left(\sin ^{2} \theta+\cos ^{2} \theta \cos ^{2} \beta\right)^{5 / 2}},
$$

(15 b)

$$
Z=\frac{\left\{\frac{g}{2} \cos \theta \cos \beta\left(\sin ^{2} \theta+\cos ^{2} \theta \cos ^{2} \beta\right)^{\frac{1}{2}}-\omega^{\frac{\sigma_{1} V_{1}-\sigma_{2}}{\sigma_{1}-\sigma_{2}}}\left(\sin ^{2} \theta+\cos ^{2} \theta \cos ^{2} \beta\right)^{-{ }^{3}-}\right\}}{2 \omega^{2}\left(\sin ^{2} \theta+\cos ^{2} \theta \cos ^{2} \beta\right)^{2}} .
$$

The angle of inclination $\alpha$ is expressed by

$$
\tan a \equiv \frac{d z}{d y}=-\frac{2 \omega \sin \theta\left(\sigma_{1} u_{1}-\sigma_{2} u_{2}\right)}{g\left(\sigma_{1}-\sigma_{2}\right)-2 \omega \cos \theta \cos \beta\left(\sigma_{1} u_{1}-\sigma_{2} u_{2}\right)} .
$$

As these expressions are a little complicated, so we treat only the case when $\beta=0$, that is, the case when there exist only zonal currents, because there exists essentially no difference between this case and the general one. Then $\varepsilon=0$ from (13), and the equations (14 a), (15 a), (15b) and (16) are fairly simplified:

$\left(14 \mathrm{a}^{\prime}\right)$

$$
p=-\frac{g \sin \theta}{q \omega^{2}}
$$

$$
\begin{aligned}
& Y=-\frac{\cot \theta}{4 \omega^{2}}+\frac{\sigma_{1} V_{1}-\sigma_{2} V_{2}}{2 \omega g \sin \theta \cdot\left(\sigma_{1}-\sigma_{2}\right)}, \\
& Z={ }_{-}^{g \cos \theta}-\frac{\sigma_{1} V_{1}-\sigma_{2} V_{2}}{2 \omega\left(\sigma_{1}-\sigma_{2}\right)},
\end{aligned}
$$

$$
\tan a=-\frac{2 \omega \sin \theta\left(\sigma_{1} u_{1}-\sigma_{2} u_{2}\right)}{g\left(\sigma_{1}-\sigma_{2}\right)-2 \omega \cos \theta\left(\sigma_{1} u_{1}-\sigma_{2} u_{2}\right)} .
$$

Putting $V_{2}=V, \quad V_{1}=V+\Delta V, \quad T_{2}=T, \quad T_{1}=T+\Delta T, \sigma_{2}=\sigma, \quad \sigma_{1}=\sigma+\Delta \sigma$, the above relations are further simplified. Now as 


$$
\frac{\Delta \sigma}{\sigma}=-n \frac{\Delta T_{0}}{T_{0}}=-\frac{g}{g-R \gamma} \frac{\Delta T_{0}}{T_{0}}
$$

( $\Delta T_{0}$ can be written as $\Delta T^{\prime}$ ), then

$$
\begin{aligned}
& Y=\frac{-g \cos \theta+2 \omega u}{4 \omega^{2} g \sin \theta}-\frac{\Delta u}{2 n \omega g \sin \theta \frac{\Delta T_{0}^{\prime}}{T_{0}^{\prime}},} \\
& Z=\frac{g \cos \theta-2 \omega u}{4 \omega^{2}}+\frac{\Delta u}{2 n \omega \frac{\Delta T_{0}}{T_{0}}},
\end{aligned}
$$

$$
\tan a=-\frac{2 \omega \sin \theta\left(u-\frac{T_{0}}{n} \frac{\Delta u}{\Delta T_{0}}\right)}{g-2 \omega \cos \theta\left(u-\frac{\Delta u}{n} \cdot \frac{T_{0}}{\Delta T_{0}}\right)} .
$$

Substituting numerical values (in CGS units):

$$
\begin{aligned}
& g=0.98 \times 10^{3}, \omega=7.3 \times 10^{-5}, R=2.9 \times 10^{6}, \gamma=5.0 \times 10^{-5} \quad(n=1.2), \\
& \theta=\frac{\pi}{4}, \quad T_{0}=280^{\circ} \mathrm{C},
\end{aligned}
$$

we get, neglecting small terms :

$$
\begin{aligned}
& \left(15 \mathrm{a}^{\prime \prime \prime}\right) \quad Y=-4.8 \times 10^{7}-2.4 \times 10^{5} \frac{\Delta u}{\Delta T}, \\
& \left(15 \mathrm{~b}^{\prime \prime \prime}\right) \quad Z=3.3 \times 10^{10}+1.6 \times 10^{8} \frac{\Delta u}{\Delta T^{\prime}}, \\
& \left(14 \mathrm{a}^{\prime \prime}\right) \quad p=-1.7 \times 10^{10}, \\
& \left(16^{\prime \prime \prime}\right) \tan a=-\frac{1.04 \times 10^{-4}\left(u-2.3 \times 10^{2} \frac{\Delta u}{\Delta T}\right)}{9.8 \times 10^{2}-1.04 \times 10^{-4}\left(u-2.3 \times 10^{2} \frac{\Delta u}{\Delta T}\right)} \fallingdotseq 2.4 \times 10^{-5} \frac{\Delta u}{\Delta T} .
\end{aligned}
$$

From $\left(16^{\prime \prime \prime}\right)$ it can be seen that the inclination of the surface of discontinuity is the steeper, the smaller the temperature difference and the larger the wind velocity difference between the air masses on both sides of the surface. This conclusion can be seen also from the equations (15) or from Fig. 2.

Now let us consider here the types of frortal surface. It is seen from (16/') that two cases can exist, the one $\frac{\Delta i b}{\Delta T^{\prime}}>0$ and the other $\frac{\Delta u}{\Delta T}<0$. As the air temperature of the air lying under is lower in general, therefore $T_{1}<T_{2}$, that is $\Delta T<0$. Thus the two cases are:

(i) $u_{2}>u_{1}$, that is, $\Delta u<0$. As in this case $\frac{\Delta u}{\Delta T^{\prime}}>0$ and $a<\frac{\pi}{2}$, therefore the frontal surface hangs down southward. This is the surface of polar front type. In the case of polar front type, the origin O (Fig. 2) becomes more and more distant from $\mathrm{O}^{\prime}$ as $\frac{\Delta u}{\Delta T}$ becomes larger, because both $|Y|$ and $|Z|$ become larger in this case. As $\varepsilon$ is constant at a definite latitude, the inclination of the front becomes thus steeper as $\frac{\Delta u}{\Delta T}$ becomes larger. 
(ii) $u_{2}<u_{1}$, that is, $\Delta u_{b}>0$. In this case the west wind velocity of the lower layer is stronger than that of the upper layer, and as $\frac{\Delta u}{\Delta T}<0$ and $\tan a<0$, the frontal surface hangs down northward. This type will be called here as equatorial front type. In this case $\mathrm{O}^{\prime \prime}$ in Fig. 2 is the origin of the coordinates $(y, z)$. In the case of equatorial front, the origion $\mathrm{O}^{\prime \prime}$ comes nearer and nearer to $\mathrm{O}^{\prime}$ as $\left|\frac{\Delta u}{\Delta T}\right|$ becomes larger, and thus the inclination of the front becomes speeper as $\left|\frac{\Delta u}{\Delta T}\right|$ becomes larger. From the equation (16 $\left.16^{\prime \prime \prime}\right)$ it must further be noticed that the inclination of the equatorial front is in general larger than that of the polar front.

\section{Mixing Layer}

Let us consider here the effect of friction. As the motion is continuous in an air-mass, the effect of friction is small and unnecessary to be considered in it. On the other hand, it is especially large at the border of air-masses, that is, on the frontal surface where the wind velocity changes discontinuously, and on the ground. Therefore, it is sufficient if we consider the effect of friction at the border of airmasses.

The effect of friction at a frontal surface can be calculated from the dynamical stability of the surface. Therefore, we calculate here the thickness of the mixing layer generated on the surface owing to dynamical instability, as was done in the previous paper. [13]

The equation (12) of a frontal surface can also be written as follows, if a line element on the surface is expressed as $d s$ (putting $\cos \beta=1$ ),

$$
\nabla\left\{\omega^{2} \sin ^{2} \theta \cdot y^{2}-2 \omega^{2} \sin \theta \cos \theta \cdot y z+\omega^{2} \cos ^{2} \theta \cdot z^{2}\right.
$$

or

$$
\left.+\omega \sin \theta \frac{\sigma_{1} V_{1}-\sigma_{2} V_{2}}{\sigma_{1}-\sigma_{2}} y+\left(\frac{g}{2}-\omega \cos \theta \frac{\sigma_{1} V_{1}-\sigma_{2} V_{2}}{\sigma_{1}-\sigma_{2}}\right) z\right\} \cdot d s=0,
$$

$\left(18^{\prime \prime}\right)$

$$
2 \omega \sin \theta \frac{\sigma_{1} u_{1}-\sigma_{2} u_{2}}{\sigma_{1}-\sigma_{2}} d y+\left(g-2 \omega \cos \theta \frac{\sigma_{1} u_{1}-\sigma_{2} u_{2}}{\sigma_{1}-\sigma_{2}}\right) d z=0 .
$$

This indicates that on the frontal surface a force

$$
\left(-2 \omega \sin \theta \frac{\sigma_{1} u_{1}-\sigma_{2} u_{2}}{\sigma_{1}-\sigma_{2}}, \quad 2 \omega \cos \theta \frac{\sigma_{1} u_{1}-\sigma_{2} u_{2}}{\sigma_{1}-\sigma_{2}}\right)
$$

acts other than the gravitational force $(0,-g)$, and that the surface is orthogonal to the composed force of the two. The absolute value $g^{\prime}$ of this composed force is

$$
\begin{aligned}
g^{\prime} & =\left\{\left(g-2 \omega \cos \theta \frac{\sigma_{1} u_{1}-\sigma_{2} u_{2}}{\sigma_{1}-\sigma_{2}}\right)^{2}+\left(2 \omega \sin \theta \frac{\sigma_{1} u_{1}-\sigma_{2} u_{2}}{\sigma_{1}-\sigma_{2}}\right)^{2}\right\}^{-\frac{1}{2}} \\
& \fallingdotseq g\left(1-\frac{2 \omega}{g} \frac{\sigma_{1} u_{1}-\sigma_{2} u_{2}}{\sigma_{1}-\sigma_{2}}\right) \fallingdotseq g\left\{1-\frac{2 \omega}{g}\left(u-\frac{T_{0}}{n} \frac{\Delta u}{\Delta T}\right)\right\} .
\end{aligned}
$$

The critical wave length $L$;

$$
L=\frac{2 \pi}{\mu} \quad \therefore(\mu: \text { wave number) }
$$


which determines the dynamical instability of frontal surfaces, is expressed [13], [15], using $g^{\prime}$ instead of $y$, by

$$
\begin{aligned}
L_{1} & =2 \pi \frac{q_{2}\left(u_{1}-u_{2}\right)^{2}}{q_{1}-q_{2}} \cdot \frac{1}{g^{\prime}}=2 \pi \frac{T_{1}}{g} \cdot \frac{\left(u_{1}-u_{2}\right)^{2}}{T_{1}^{\prime}-T_{3}^{\prime}}\left(1+\frac{2 \omega}{g} \frac{\sigma_{1} u_{1}-\sigma_{2} u_{2}}{\sigma_{1}-\sigma_{2}}\right) \\
& =\frac{2 \pi^{\prime} T^{\prime}}{g} \frac{(\Delta u)^{2}}{\Delta T^{\prime}}\left\{1+\frac{2 \omega}{g}\left(u-\frac{T_{0}}{n} \frac{\Delta u}{\Delta T^{\prime}}\right)\right\}
\end{aligned}
$$

Substituting numerical values, we get

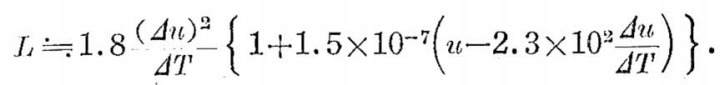

It is seen from this equation that the thickness of the mixing layer developed on a frontal surface is the larger, the larger the wind velocity difference and the smaller the temperature difference on both sides of the surface. Combining this conclusion with that derived from the equation $\left(16^{\prime \prime \prime}\right)$, we can say that the thickness of the mixing layer. generated on a frontal surface is the larger, the steeper the surface inclines. Here we can

\begin{tabular}{|c|c|c|c|c|}
\hline $\begin{array}{l}\Delta, t \\
\Delta t \\
(\mathrm{o} C)\end{array}$ & 100 & 40 & 20 & 10 \\
\hline 1 & $\begin{array}{l}1,170 \\
2,400\end{array}$ & $\begin{array}{l}250 \\
350\end{array}$ & $\begin{array}{l}67 \\
77\end{array}$ & $\begin{array}{l}17 \\
19\end{array}$ \\
\hline 2 & $\begin{array}{r}750 \\
1,050\end{array}$ & $\begin{array}{l}136 \\
153\end{array}$ & $\begin{array}{l}35 \\
37\end{array}$ & 9 \\
\hline 4 & $\begin{array}{l}410 \\
490\end{array}$ & $\begin{array}{l}69 \\
75\end{array}$ & 18 & \\
\hline 10 & $\begin{array}{l}170 \\
180\end{array}$ & 29 & & \\
\hline
\end{tabular}
interpret the thickness as the horizontal extent of the layer.

Table. Thickness of the Mixing I ayer (in km).
Now we calculate the thickness $L$ of the mixing layer. The result is shown in the table, where numerical values of $L$ (in $\mathrm{km}$ ) are calculated corresponding the various values of $\Delta u$ (in $\mathrm{m} / \mathrm{s}$ ) and $\Delta T$ (in ${ }^{\circ} \mathrm{C}$ ). The value of $u$ is taken $u=100 \mathrm{~m} / \mathrm{s}$. The upper figures correspond to the polar front and the lower

ones to the equatorial front respectively.

From the table it is seen that the thickness often reaches to the order of $1,000 \mathrm{~km}$. It is futher noticeable that the thickness becomes rapidly larger as the wind velocity difference becomes larger.

\section{Existence of the Jet Stream}

In order to know strictly the motion of the atmosphere, we must further integrate the intermediate integrals (4), (5) and $(6)$, or $\left(4^{\prime}\right),\left(5^{\prime}\right)$ and $\left(6^{\prime}\right)$, or $\left(4^{\prime \prime}\right),\left(5^{\prime \prime}\right)$ and $\left(6^{\prime \prime}\right)$. As it is very difficult to do so, we will here interpret the whole character of the motion, starting from a simple example.

Putting $s=$ const. $(=1), \omega=0$ and expanding $r^{r}(\psi)$ and $\Psi^{\prime}(\psi)$ in terms of $\psi$, we get from the equations $\left(4^{\prime \prime}\right)$ and $\left(5^{\prime \prime}\right)$

$$
\text { (22 a), (22 b) } \quad u=a \psi, \quad \frac{\partial^{2} \psi}{\partial y^{2}}+\frac{\partial^{2} \psi}{\partial \varepsilon^{2}}=\left(b^{2}-a^{2}\right) \psi .
$$

As $\phi=$ const. $\left(=\psi_{0}\right)$ on the boundary surface, so we can easily get a solution from 
(22) which expresses a convective motion in the $y-z$ plane, if the form of the boundary is simple [12]. In this case the equation (22 b) gives the value of $\psi$, that is, a convective motion in the $y-z$ plane and $(22$ a) gives the value of $u$ as a function of $\psi$.

Also in the case when the form of the boundary is not simple, we can infer the state of motion which the equations (22) express, because the streamlines must always be closed and almost parallel to the boundary in its neighbourhood.

The same can be said as to the current in the $y-z$ plane which the equation $\left(5^{\prime \prime}\right)$ expresses. In the case of $\left(5^{\prime}\right)$, the effects of $s$ which varies mainly with altitude and of the deflecting force come into consideration, but it is the same as above that the streamlines expressing a convective motion are closed and almost paralle 1to each other, corresponding to the form of the boundary.

Further, as the isovels of the $x$-component $u$ of velocity coincide with the streamlines in the $y-z$ plane, the three-dimensional motion which (22) express is the ofe which moves in a spiral-like shape on a cylinder-surface, the section of which is expressed by $\phi=$ const.

The motion which the equaions (4) and (5), or $\left(4^{\prime}\right)$ and $\left(5^{\prime}\right)$ express is almost the same. Only it must be noticed in this case that the absolute anglar momentum $r \sin \theta \cdot v+\omega r^{2} \sin ^{2} \theta$ is conserved along a streamline $\psi=$ const. in the meridional plane, and the wind velocity $v$ itself varies as

$$
v=\frac{V}{r \sin \theta}-\omega r \sin \theta
$$

Thus in this case the isovels of $v$ do not coincide with the streamlines in the meridional plane (projection). As the effect of variations of $V(\psi)$ is considered to be relatively small, it can be easily seen that, if air particles of lower latitudes move northward conserving angular momentum, then they must get extremely strong west wind components. Thus it is clear that, at least in an air-mass, air particles moving convectively in the meridional projection get strong in west wind velocity as they approach to the pole, and do so in east wind velocity as they go awaig from the pole.

On the basis of the above mentioned idea, we consider here the state of motion in the troposphere.

In the neighbourhood of the pole $V$ must be zero, in order that $v$ does not become infinitely large at the pole. That is, the absolute angular momentum must be zero in the polar air-mass in order that a motion conserving angular momentum can exist there. Therefore

$$
v=-\omega r \sin \theta \text {. }
$$

This indicates a motion which is standing still like a solid body relative to the absolute spacf. When this motion is observed from the rotating earth, the motion is such that in the polar region there blow easterly winds in proportion to the axial distance. Thus the velocity in the polar east wind region becomes larger south. ward.

The form of the polar tropopause is expressed approximately by 


$$
r=\frac{\sigma_{1} \Psi_{1}-\sigma_{2} \Psi_{2}}{g\left(\sigma_{1}-\sigma_{2}\right)}-\frac{\omega^{2}}{2 g} r^{2} \sin ^{2} \theta
$$

if we can consider that $V$ is also zero in the part of the stratosphere, which also contains the polar axis. Thus, in the extent that we can consider the earth as a sphere, the polar tropopause has a form approximate to a parabola.

In reality, as there exists the effect of friction in the polar air-mass and especially in the stratosphere, the relations (24) and (25) will be altered more or less. Thus the wind velocity (24) will be weakened and there may exist a convective motion balancing with friction in the polar air-mass and also in the stratosphere.

It is not strict to use equation $\left(11^{\prime \prime}\right)$ or (12) for the determination of the shape of tropopause, because in the stratosphere temperature either decreases upward little or often increases. As, however, the difference between the shapes derived from the equations (11') and $\left(11^{\prime \prime}\right)$ respectively is small, therefore we proceed here with discussion using the approximate relation $\left(11^{\prime \prime}\right)$. In reality, the air in the lower stratosphere is also drawn down, when the tropopause is sucked down by strong winds in the troposphere (as will be stated later). Then a temperature lapse-rate almost equal to that in the troposphere appears in the lower part of the stratosphere[13]. In this case the equation (12) can be used sufficiently strictly.

The shape of the polar front and that of the equatorial tropopause are to be derived from the equation $\left(11^{\prime}\right)$ or $\left(11^{\prime \prime}\right)$. But it is sufficiert if we use here relations derived from (12).

As has been shown before, there exists in the troposphere on the south of the polar front a convective motion in the meridional plane having streamlines almost parallel to the earth's surface, to the polar front surface and then to the equatorial tropopause (meridional circulation). As the absolute anguler momentum is conserved $(V \neq 0)$ in this motion, the west wind velocity is extremely strong at the northern end (the triple point between the stratosphere, the polar troposphere, and the southern troposphere) of this air-mass, and it sucks up the polar front and sucks down the equatorial tropopause. That is, the air from lower latitudes conserving angular momentum can not proceed on its way over some extent northward, as it makes itself a barrier by drawing the surfaces of discontinuity toward itself. Thus at the triple point the inclinations of the polar front as well as of the equatorial tropopause are fairly steep.

On the other hand, the steeper the inclination of frontal surface, the larger the thickness of the mixing layer becomes and it often reaches to the order of $1,000 \mathrm{~km}$. Thus in the northern part of a tropopspheric air-mass bordered by tne surfaces of discontinuity, there must exist a portion where the west wind velocity is extremely strong. This portion where the wind velocity is maximum siluates strictly at the northern end of the southern side of the mixing layer. The wind must be considered to be the jet stream.

Thus it is seen that the jet stream is generated, because there exist motions in the atmosphere conserving angular momentum, and the motion can not stretch over some extent 
northward owing to the surfaces of discontinuity which are drawn near by it. The effect of friction in this case is to shift the position of the jet stream southward and diminish the maximum value of wind velocity.

\section{Structure of the Jet Stream}

Now we can describe the structure of the jet stream thus derived. Three surfaces of discontinuity, that is, the polar tropopause (PT-T), the equatorial tropopause (ET-T) and the polar front ( $\mathrm{PF}-\mathrm{T}$ ) encounter each other at the triple point $T$ (see Fig. 3). Among these surfaces the inclination is the smallest at the polar

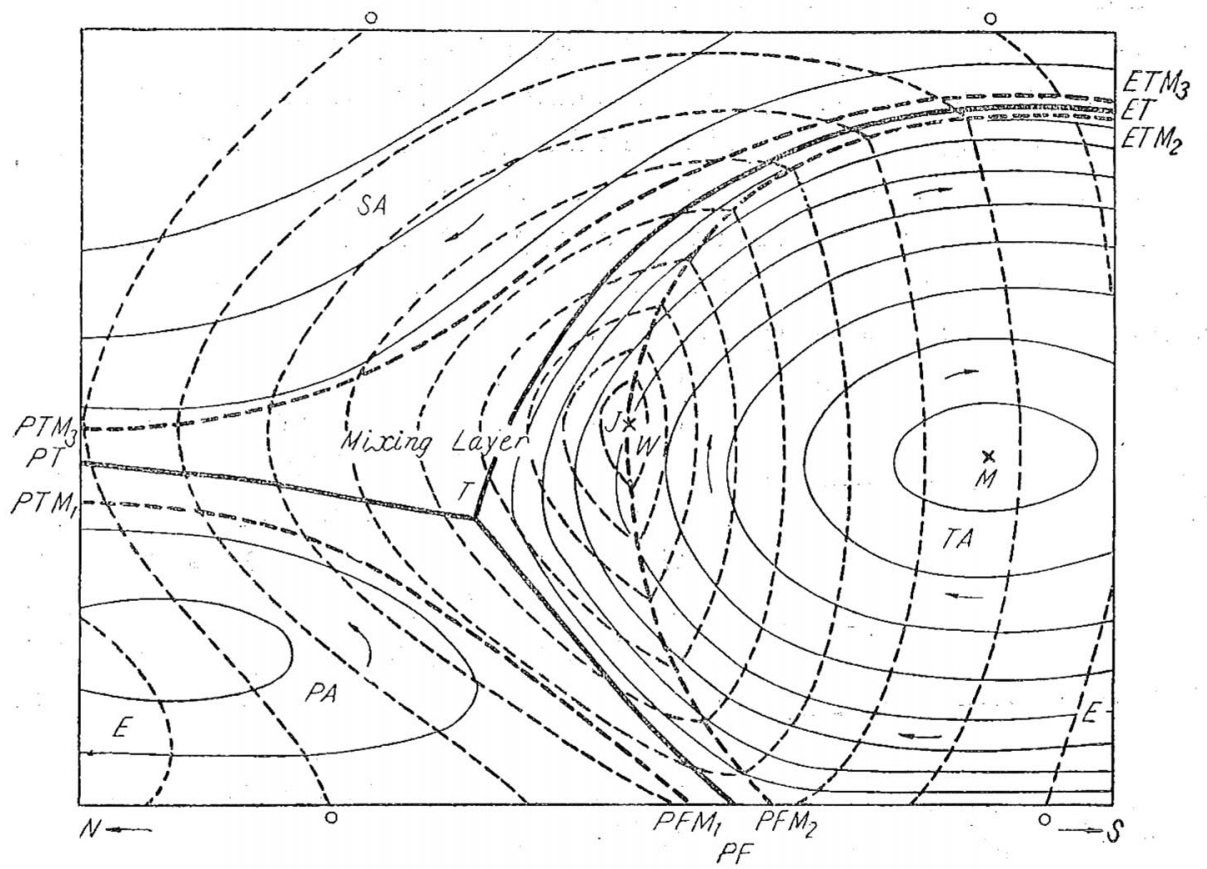

Fig. 3. Meridional Section of a Jet Stream.

Heavy full lines indicate initial surfaces of discontinuity, heavy broken lines side surfaces of the mixing layer, thin full lines streamlines in the meridional plane, thin broken lines isovels of zonal wind.

tropopause and the largest at the equatorial tropopause; because the temperature difference is smaller (the temperature is higher in the stratosphere, because the stratospheric air just at the north of the equatorial tropopause is sucked down and is warmed almst adiabatically) and the wind velocity difference is fairly larger (in the stratosphere the law of conservation of momentum does not hold and the wind seems to be in equilibrium with friction, therefore the wind there can not be stronger than that in the jet stream) at the equatorial tropopause than at the polar one. The inclination of the polar front is smaller than that of the equatorial tropoqause.

The motion is the most developed in the tropical troposphere (TA). Along a streamline is conserved absolute angulare momentum. In actual cases $V(\psi)$ is 
considered to increase with the decrease of $\psi$ ( $\psi$ is now considered to be minimum in the middle of the troposphere), therefore the isovels of west wind are almost parallel to earch other and swell southward a littile.

In the polar troposphere (PA), the east wind is fairly weakened in the southern part, and often west winds may dominate there owing to friction. Therefore the maximum of the east wind may deviate fairly northward from the polar front.

In the stratosphere (SA), it is considered that a slow northward current and a west wind accompanying it exist in the lower layers, which seem to be rather weakened northward up to zero at the pole.

The thickness of the mixing layer developed at the fronts is the largest at the lower part of the equatorial tropopause and it often reaches to the order of $1,000 \mathrm{~km}$.

The wind velocity is smoothed in the mixing layer, and therefore the vorticity (the ver tical component of) becomes constant, if the layer reaches to the pole. In actual cases it holds also approximately, as the vorticity is constant in the polar air-mass. Thus we can say that the contancy of absolute angular momentum holds approximately southward, and the constancy of absolute vorticity holds approximately north-
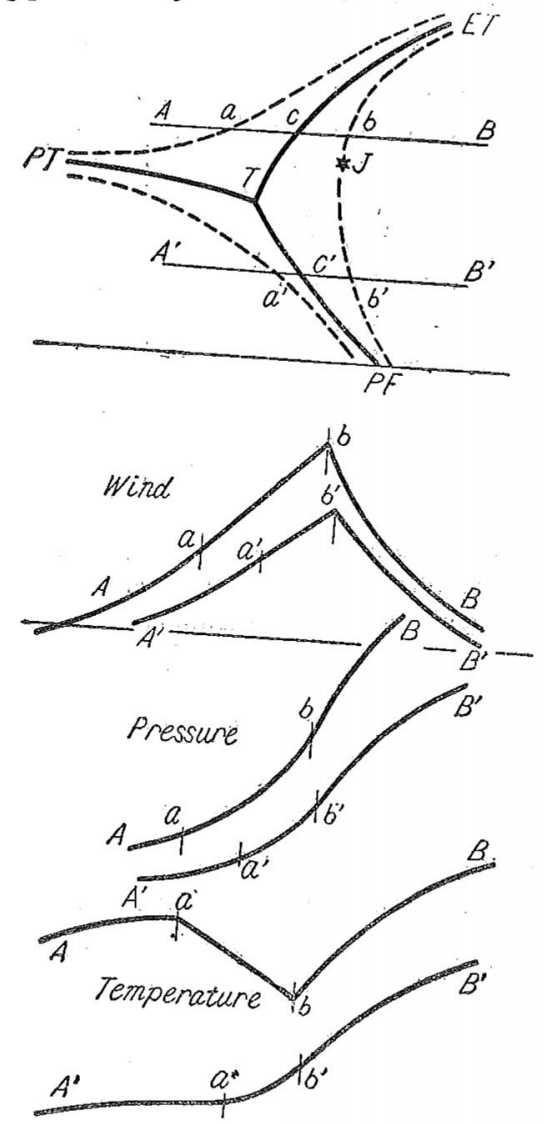

Fig. 4 Distribution of Wind, Pressure and Temperature along Meridional Horizontal Lines. ward from the southern ride of the mixing layer, where the west wind velocity is maximum.

As the effet of friction is large both in the stratosphere and in the polar troposphere, the upper side $\left(\mathrm{PTM}_{3}-\mathrm{EFM}_{3}\right)$ and the porthen side $\left(\mathrm{PFM}_{1}-\mathrm{PTM}_{1}\right)$ of the mixing layer are not distinct and only the southern side $\left(\mathrm{ETM}_{2}-\mathrm{J}-\right.$ $\mathrm{PFM}_{3}$ ) will be clearly observed. Thus, it will be difficult to analyse precisely the front in the neighbourhood of the jet. stream $J$ or the triple point $\mathrm{T}$.

Next we consider the distribution of pressure. In an air-mass, pressure is expressed as (26) $p^{\frac{R}{g} r} \fallingdotseq \sigma\left\{\Psi-g r-\frac{1}{2}\left(\frac{V}{r \sin \theta}-\omega r \sin \theta\right)^{2}\right\}$. Thus the isobaric surface inclines northward in most cases. This result holds also approximately for the stratosphere, but is not strict owing to the effect of friction. As $V=0$ in the polar air-mass, the pressure theie becomes higher towards the pole. Also in the stratosphere, $V$ can be considered almost zero in the neighbourhood of the pole, therefore the pole may be the center of high pressure also in the stratosphere. In the mixing layer, the pressure curve shows itself as it shifts gradually and 
smoothly to the pressure profile of each air-mass [13], and the pressure gradient is the steepest at the southern side of the mixing layer. Thus the pressure distribution becomes as is shown in Fig. 4.

In the equatorial troposphere, isothermal surfaces incline northward with isobaric surfaces. Those in the stratsphere also incline slowly northward. As the air in the lower stratosphere is warmer than that in the equatorial troposphere, the temperature increases not thward in the mixing layer $a b$. The temperature decrease is not remarkably weakened by mixing at the polar front, so the temperature discontinuity will be easily analysable there.

The auther discussed the structure of typhoon before [13], and pointed out the existence of a maximum of wind velocity, that is, a sort of jet stream at the outer side of the tropopause funnel forming the eye of typhoon, strictly speaking, at the lower part of the outer side of the mixing layer. The existence of the jet stream is considered thus to be a common phenomenon in all the motions conserving angular momentum.

\section{On the Mechanism of the General Circulation}

The main problem of the general circulation is considered to explain the mean state of the atmosphere. Then, to discuss it one is to start from the equations of motion with friction, or preferably from the equations of mean motion, because friction has especially important meanings in the general circulation.

As, however, there exist fairly predominant and systematic perturbations in the atmosphere, it may miss important characteristics accompanying the general circulation, if one persists only on the mear state. Therefore the author intends here to discuss the state of the general circulation in the case when the relations concerning stationary motion hold and the effect of friction is considered sufficient if it is applied only at the border surface.

The air, for instance, in middle latitudes conserving angular momentum takes a velocity of about $100 \mathrm{~m} / \mathrm{s}$ by moving a distance of $10^{\circ}$ latitudes northward even if its initial velocity is $0 \mathrm{~m} / \mathrm{s}$. Then, the strong winds generated by meridional convection draw near the surfaces of discontinuity from both upper and lower layers and form themselves a barrier. Thus, in the limits that the effect of friction is considered to be secondary, the meridional circulation of the troposplere must have a cellular. structure, and a pair of jets is generated as a r'ule in each cell, among which only the wrst wind jet is predominant.

At the border of each cell, momentum is transferred northword only by the effect of friction. Thus, in the case when the troposphere is divided into several cells, the mean absolute momentum of each cell decreases gradually from the tropical cell to the polar cell, at which the absolute angular momentum is zeio. The mean west wind velocity itself of each cell becomes, however, larger northward except of the polar cell, but it does not become so strong as in the case when the whole troposphere is supposed to be one cell.

In the tropical cell, the mean wind may be considered to be directed eastward. 
Then in the southern half part of this cell, especially in the upper layers there blows easterly wind, and in the northern half part there blows westerly wind, owing to which the tropopause hangs down northward. The convection in the tropical cell is such that the warmed air near the equator ascends, moves northward along the inclined tropopause and then descends along the border with the neighbouring cell, and a west wind jet will be found also at the northern part of this tropical cell.

The meridional circulation in the cell in middle latitudes is directed northward near the earth's surface and southward along the tropopause, and thus a remarkable west wind jet will be found near the polar front. As the polar cell is dynamically rest$\operatorname{rained}(V=0)$, and the west wind velocity is considerably strong in the cell in middle latitndes, therefore perturbations seem to be the most predominant at the polar front. Each cell will fluctuate thus in the neighbourhood of the mean position. The figure, in which the jets, especially the northern one, are smoothed, is now widely adopted as the ordinary model of the general circulation [15].

As we treated a stationary problem, we can say nothing certain on the action intensifying or weakening the jet stream, nor on its fluctuation. We discussed only on jits structure.

Acknowledgement - As the author is not surfficiently informed in recent papers, more suitable ones may possibly befound other than the references cited. The author expects the tolerance and kind instruction of the readers on this point. Further, thanks are devoted to Mr. T. Watanabe who assisted the author in preparing the references.

\section{Heferences}

[1] Rossby,C.G.,1947: On the Distribution of Angulay Velocity in Gaseous Envelops under the Influence of Large Scale Horizontal Mixing Processes, Bull. Aıer. Met. Soc., 28.

[2] Staff Membens of the Dep'r. of Met., Univ. of Chicago, 1947: On the General Circulation of the Atmosphere in Middle Latitudes, Bull. Amer. Met. Soc., 28.

[3] Namtas, J.,1947: Physical Nature of Some Fluctuations in the Speed of the General Circulation, Journ, Met. , 4.

[4] Irrenl, H., 1948: Jet Stream in Upper Troposphere and Cyclone Formation, Trans. Amer. Geophys. Union, 29.

[5] Namras, J., and P. F. CunpP, 1949: Confluence Theory of the High Tropospheric Jet Strean, Jonm. Met. , 6.

[6] Namras, J., 1950: The Index Gycle and its Role in the General Circulation, Journ. Net. 7 .

[7] Gressman, G.P., 1950: Variation in the Structure of the Upper Westerlies, Jour. Met., 7 .

[8] PALMf́, E., 1948: On the Distribution of Temperature and Wind in the Upper IVesterlies, Journ. Met. , 5 .

[9] Palmín, E. and K. M. NAGLer, 1948: An Analysis of the Wind and Temperature Distribution in the Free Atmosphere over North Anerica in a Case of Approximately Westerly Flow, Journ. Met., 5.

[10] Patmis, E. and C.W. Newton, 1948: A Study of the Mean Wind and Temperature Distribution in the Vicinity of the Polar Front in Winter, Journ. Met., 5.

[11] Riehl, H., J.G. YEN and N.E. JA Seur, 1950: A Study of Variations of the 
General Circulation, Journ. Met., 7 .

[12] Sato, T., 1950: Structure of the Stationary Discontinuous Sirface, (1), (2) and (3), Journ. Met. Soc. Japan, 28.

[13] Sato, T., 1951: On the Structure of Typhoon, Geophss. Mag., (yet unpudlished)

[14] SAто, T., 1951: On the Equations of Stationary Motion, Papers in Met. and Geophys., II.

[15] Bjerkness, V., and Colraboratos, 1933: Physikalische Hydrodynamik.

\section{Discussion}

Question: We have carefully discussed the foregoing paper "Dynamics of the Jet Stream" by Mr. T. SATO with much interest. We think that the analytic condition for the tropopause given by $\left(11^{\prime \prime}\right)$ is far from the current conception, whereas (11/') stands for the frontal surface (Discontinuitätsfläche nullter Ordnung), assuming the same lapse-rate in the troposphere and the stratosphere, and so the analytic condition for the tropopause should be different from (11'I).

\section{H. Arakawa}

Reply: The auther wishes to express his hearty thanks to Dr. H. ARAKA wa and his collaborators for their detailed discussions on the present pajer.

We did not touch the conditions for the tropopause in this paper, as they were partly discussed in the preceding paper "On the Structure of Typhoon," the publication of which is rather delayed.

We want to study the surace of discontinuity (including the tropopause) dynamically as well as thermally. It is considered then that the effect of friction is especially large at the surface of discontinuity, but, as the strict integration of the equations of motion of frictional filuid is guite hopeless for the present, it would be the only way we can do at present to consider the motion of frictionless fluid as a first approximation, and then to add the effect of triction at places where it is especially predominant (at the internal surface of discontinuity and at the groumd), that is, to consider only the effect of external friction. The present paper followed this course.

As it is clear that convective motions (such as typhoons, cyclones, etc.) in the troposphere can not penetrate through the stratosphere, it follows that there exists a surface of discontinuity between them, and it seems to us that this surface is also satisfied by (11) derived from the dynamical boundary condition, in the extent that the effect of friction is negligible. The actual tropopause as well as the frontal surfaces in the troposphere seems to us to be diffused owing to the effect of friction, as is shown in section 4 and later in the paper.

After our treatment, the velocity $v$ and the dencity $q$ (or the temperature $I^{r}$ ) are continuous on the side-surfaces of the mixing layer, the normal components of their dreivatives $\frac{\partial v}{\partial n}$ and $\frac{\partial q}{\partial n}$ ( or $\frac{\partial T}{\partial n}$ ), however, become discontinuous on the surfaces (see Fig. 4).

Althongh the state of the stratosphere is different thermally and dynamically from tlat of the troposphere, it seems thus that the tropopause, seen as a surface of discontinuity, is not essentially different from the surfaces of discontinuity in the troposphere.

It is desirable, in this sense, to analyse the atmospheric suriaces of discontinuity and the associated phenomena nore precisely in dynamical way. 